H. Sato

Nagoya Math. J.

Vol. 60 (1976), 81-91

\title{
CUSPS ON BOUNDARIES OF TEICHMÜLLER SPACES
}

\author{
HIROKI SATO
}

\section{Introduction.}

We shall be concerned here with cusps on the boundaries of Teichmüller spaces.

Bers [3], Maskit [7] and Kalme [5] studied about boundaries of Teichmüller spaces $T(\Gamma)$. According to Bers [3], the boundary of $T(\Gamma)$ consists of cusps and degenerate groups. Maskit [7] showed how to construct b-groups. Furthermore Kalme [5] concretely constructed cusps in the case of genus 1 by "pinching" and "twisting" deformations. At first we consider the subspace of $T(\Gamma)$ which consists of hyperelliptic surfaces. By means of variation of distributions of branch points we consider a deformation of Riemann surfaces. We shall show that, when the distance between two branch points tends to zero, the sequence of points in $T(\Gamma)$ represented by the deformation approaches to a cusp on the boundary $\partial T(\Gamma)$ (Theorem 1). For general Teichmüller space $T(\Gamma)$, where $U / \Gamma$ is a compact Riemann surface of genus $g, g \geqq 2$, we shall show that by the "pinching and twisting" deformation (not Dehn's twisting, see $\S 3$ for the definition), we get a sequence of points in $T(\Gamma)$ which approach to a cusp on $\partial T(\Gamma)$ (Theorem 2). For the proof of Theorems 1 and 2, we do not use an "inequality of Bers" (Bers [3]) but use the method of extremal length. Theorem 1 is essentially known (Bers [3]) but the author does not know the theorem stated in this style. The important thing is that the proof of the theorem is applicable not only to the case of cusps on $\partial T(\Gamma)$ but also to the case of cusps on "boundaries" of Schottky spaces and of spaces of regular b-groups.

In $\S 1$ we shall give the terminology and definitions. We shall study in $\S 2$ the cusps on $\partial T(\Gamma)$ obtained by "pinching" deformation and in $\S 3$ the cusps on $\partial T(\Gamma)$ obtained by "pinching and twisting" deformation.

The author wishes to express his gratitude to Professors K. Oikawa

Received February 20, 1975. 
and $\mathrm{Y}$. Miyahara for their perseverance in criticizing preliminary draft of this paper, and to Professor K. Matsumoto for bringing the problem of Theorem 1 to his attention several years ago.

\section{Preliminaries and terminology.}

For the latter necessity we shall state the definition of spaces of Kleinian groups due to Kra [6]. In order to treat the spaces of Kleinian groups, we represent the elements in the space by Beltrami coefficients. We denote by $S L^{\prime}(2, C)$ the space of Möbius transformations, that is, an element $A$ in $S L^{\prime}(2, C)$ is represented by $A(z)=(a z+b)$ $/(c z+d), z \in \hat{C}(=C \cup\{\infty\})$ or a matrix $A=\left(\begin{array}{ll}a & b \\ c & d\end{array}\right) \in S L^{\prime}(2, C)$ modulo $\{ \pm 1\}$. Especially we denote the space by $S L^{\prime}(2, R)$ instead of $S L^{\prime}(2, C)$ in the case of $a, b, c, d \in R$. Let $G \subset S L^{\prime}(2, C)$ be a Kleinian group, and let $\Delta$ be an invariant union of connected components of $\Omega=\Omega(G)$, the region of discontinuity of $G$. Denote by $\Lambda=\Lambda(G)$ the limit set of $G$.

A Beltrami coefficient $\mu$ for $G$ is a measurable function satisfying

(1) $\mu(A z) \overline{A^{\prime}(z)} / A^{\prime}(z)=\mu(z), A \in G$, a.e. $z \in \Omega$

(2) $\mu \mid \Lambda=0$

(3) $\operatorname{esssup}|\mu|<1$.

The Beltrami coefficients form the open unit ball of the Banach space of bounded measurable functions on $\Omega / G$. The space of Beltrami coefficients for $G$ with support in $\Delta$ is denoted by $M(G, \Delta)$. It is well known that for every $\mu \in M(G, \Delta)$ there is a unique q.c. automorphism $w^{\mu}$ of $\hat{C}$ satisfying the Beltrami equation $\left(w^{\mu}\right)_{z}=\mu\left(w^{\mu}\right)_{z}$ and $w^{\mu}(0)=0$, $w^{\mu}(1)=1$ and $w^{\mu}(\infty)=\infty$ (hereafter we write $w^{\mu}(0,1, \infty)=(0,1, \infty)$ ). For fixed $A \in G$ and $\mu \in M(G, \Delta)$, we have

$$
w^{\mu} A\left(w^{\mu}\right)^{-1} \in S L^{\prime}(2, C)
$$

Thus each $\mu$ determines an isomorphism called a quasi-conformal deformation of $G$

$$
\theta(\mu): G \rightarrow S L^{\prime}(2, C)
$$

where $\theta(\mu)(A)$ is given by (1). We call $\mu \in M(G, \Delta)$ trivial if (2) is the identity isomorphism. The set of trivial Beltrami coefficients for $G$ with support in $\Delta$ is denoted by $M_{0}(G, \Delta) . \quad \mu$ and $\nu \in M(G, \Delta)$ are called equiv- 
alent if $\theta(\mu)=\theta(\nu)$. The set $M_{0}(G, \Delta)$ acts as a group of right transformation on $M(G, \Delta)$ by

$$
M(G, \Delta) \times M_{0}(G, \Delta) \ni(\mu, \nu) \mapsto \mu \nu \in M(G, \Delta),
$$

where $w^{\mu \nu}=w^{\mu} w^{\nu}$. In this manner, we view $M_{0}(G, \Delta)$ as a group of biholomorphic automorphisms of $M(G, \Delta)$. The quasi-conformal deformation space of $G$ with support in $\Delta$ is

$$
T(G, \Delta)=M(G, \Delta) / M_{0}(G, \Delta)
$$

endowed with the quotient topology. If $\Gamma$ is a Fuchsian group operating on $U$, the upper half plane, then $T(\Gamma, U)=T(\Gamma)$ is the usual Teichmüller space if and only if $\Gamma$ of the first kind. From now on $\Gamma$ denotes a finitely generated Fuchsian group of the first kind.

Let $\mu \in M(\Gamma, U)$. Then there exists an unique q.c. automorphisms $w_{\mu}$ of $U$ that satisfies the Beltrami equation $\left(w_{\mu}\right)_{z}=\mu\left(w_{\mu}\right)_{z}$ and the normarization $w_{\mu}(0,1, \infty)=(0,1, \infty)$. Every $\mu \in M(\Gamma, U)$ determines an isomorphism defined by setting $\theta(\mu) A=w_{\mu} A W_{\mu}^{-1}, A \in \Gamma$. Then as in the above there uniquely exists $w^{\mu}: \hat{C} \rightarrow \hat{C}$ satisfying $\left(w^{\mu}\right)_{\bar{z}}=\mu\left(w^{\mu}\right)_{z}$ and $w^{\mu}(0,1, \infty)=(0,1, \infty)$. We define a quasi-Fuchsian group $\theta(\mu)(\Gamma)=$ $w^{\mu} \Gamma\left(w^{\mu}\right)^{-1} \subset S L^{\prime}(2, C)$. Set $w^{\mu} \mid L=W_{\mu}$, where $L$ is the lower half plane. Then $W_{\mu}$ is a conformal mapping. We denote by $\left\{W_{\mu}, z\right\}$ the Schwarzian derivative

$$
\left\{W_{\mu}, z\right\}=\left(W_{\mu}^{\prime \prime} / W_{\mu}^{\prime}\right)^{\prime}-(1 / 2)\left(W_{\mu}^{\prime \prime} / W_{\mu}^{\prime}\right)^{2}, \quad z \in L .
$$

Then $\left\{W_{\mu}, z\right\}=\phi_{\mu}, z \in L$ is an element in $B(L, \Gamma)$, which is the space of bounded automorphic forms of weight $(-4)$ for $\Gamma$ in $L$. If $\mu_{1}$ and $\mu_{2}$ are equivalent under $M_{0}(\Gamma, U)$, then $W_{\mu_{1}}=W_{\mu_{2}}$ on $R$. Then $W_{\mu_{1}}\left|L=W_{\mu_{2}}\right| L$, since $W_{\mu_{1}}^{-1} W_{\mu_{2}}$ is conformal. Thus $\mu_{1}$ and $\mu_{2}$ determine the same $\phi \in B(L, \Gamma)$ Furthermore we see easily that the mapping constructed in the above from $M(\Gamma, U)$ to $B(L, \Gamma)$ is injective. Conversely, for $\phi \in B(L, \Gamma)$ we consider the linear differential equation

$$
2 \eta^{\prime \prime}(z)+\phi(z) \eta(z)=0, \quad z \in L .
$$

Let $\eta_{1}(z)$ and $\eta_{2}(z)$ be two linearly independent solutions of (4). Set $W_{\phi}$ $=\eta_{1} / \eta_{2}$ and the consider the only $\phi \in B(L, \Gamma)$ such that $W_{\phi}$ is a conformal mapping onto the one of the complements of the simple closed curve. Set $\theta_{\phi}(\Gamma)=W_{\phi} \Gamma W_{\phi}^{-1}$. Then by Bers [3] there exists a q.c. mapping 
$w_{\phi}: \hat{C} \rightarrow \hat{C}$ such that $w_{\phi} \Gamma w_{\phi}^{-1}=\theta_{\phi}(\Gamma)$ and $w_{\phi} \mid L=W_{\phi}$. We set $\mu_{\phi}=\left(w_{\phi}\right)_{z}$ $/\left(w_{\phi}\right)_{z}, z \in U$, then $\mu_{\phi} \in M(U, \Gamma)$. Let $\hat{w}_{\phi}: \hat{C} \rightarrow \hat{C}$ be another such mapping and set $\hat{\mu}_{\phi}=\left(\hat{w}_{\phi}\right)_{z} /\left(\hat{w}_{\phi}\right)_{z}, z \in U$. Then it is easily seen that $\theta\left(\mu_{\phi}\right)=\theta\left(\hat{\mu}_{\phi}\right)$. For the $\mu_{\phi}$, we determine $w^{\mu_{\phi}}$ by the same method as above first half. Setting $w^{\mu \phi} \mid L=W_{\mu \phi}$, we have $\left\{W_{\mu_{\phi}}, z\right\}=\phi$. Hence we may consider $T(\Gamma)$ as the subspace of $B(L, \Gamma)$, that is, the space of $\phi \in B(L, \Gamma)$ such that $W_{\phi}$ is the restriction to $L$ of a q.c. self-mapping $w$ of $\hat{C}$ compatible with $\Gamma$. Thus we can consider $\partial T(\Gamma)$, the boundary of Teichmüller space $T(\Gamma)$, in $B(L, \Gamma)$. For $\phi \in \partial T(\Gamma)$, we may define $\theta_{\phi}(\Gamma)=W_{\phi} \Gamma W_{\phi}^{-1}$ as in the above. Every group of the form $\theta_{\phi}(\Gamma), \phi \in \partial T(\Gamma)$ (or conjugate to such a group in $S L^{\prime}(2, C)$ ) will be called a boundary group of $\Gamma$. A point $\phi \in \partial T(\Gamma)$ is called a cusp if there is a hyperbolic element $A \in \Gamma$ such that $\theta_{\phi}(A)$ is parabolic. A point $\phi \in \partial T(\Gamma)$ is degenerate if $\theta_{\phi}(\Gamma)$ is a degenerate group, i.e., $\Omega\left(\theta_{\phi}(\Gamma)\right)$ is connected and simply connected. Bers [3] showed that $\partial T(\Gamma)$ consists of cusps and degenerates. We shall discuss cusps on $\partial T(T)$ in $\S 2$ and in $\S 3$.

\section{Cusps obtained by the "pinching" deformations.}

In this section we shall consider cusps on the boundary of Teichmüller space $T(\Gamma)$ obtained by the "pinching" deformations.

Let $S$ be the normalized hyper-elliptic surface with branch points $a_{1}, \cdots, a_{2 g-2}, 0,1, a_{2 g-1}, \infty\left(a_{1}<a_{2}<\cdots<a_{2 g-2}<0<1<a_{2 g-1}: a_{2 g-1}>\left|a_{1}\right|, a_{j} \in \boldsymbol{R}\right.$ $(j=1, \cdots, 2 g-1))$ and branch cuts $\left(a_{1}, a_{2}\right), \cdots,\left(a_{2 g-3}, a_{2 g-2}\right),(0,1),\left(a_{2 g-1}, \infty\right)$ on $R$ as in Fig. 1.

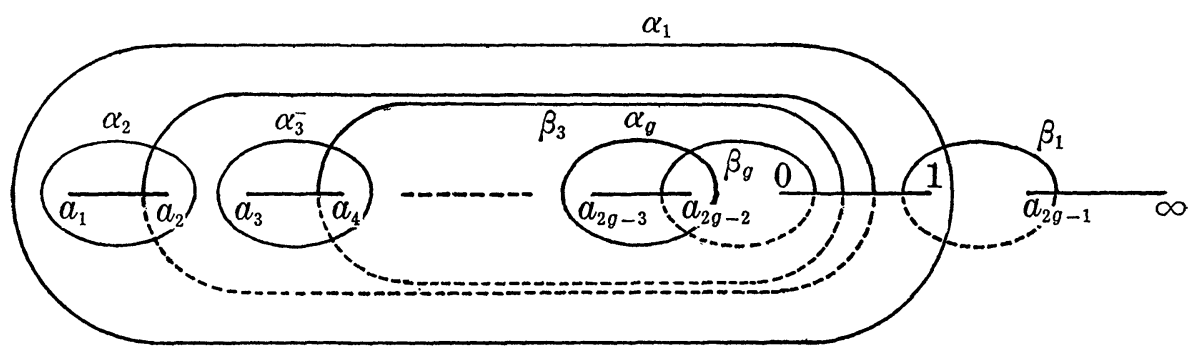

Fig. 1.

As in Fig. 1, take a homology basis $\alpha_{1}, \cdots, \alpha_{g}, \beta_{1}, \cdots, \beta_{g}$ on $S$. Now we consider the deformation under which these branch points except $a_{2 g-1}$ and these branch cuts except $\left(a_{2 g-1}, \infty\right)$ are fixed and the point $a_{2 g-1}$ tends to $\infty$. We shall consider the variation of Teichmüller points, especially the limit of the sequence of the points under this deformation. 
Let $\Gamma$ be a finitely generated Fuchsian group such that $U / \Gamma$ is the above Riemann surface $S$ and let $S_{n}$ be the Riemann surface with branch points $a_{1}, \cdots, a_{2 g-2}, 0,1, a_{2 g-1}^{(n)}, \infty$ and branch cuts $\left(a_{1}, a_{2}\right), \ldots$, $\left(a_{2 g-3}, a_{2 g-2}\right),(0,1),\left(a_{2 g-1}^{(n)}, \infty\right)$ on the real axis, where $a_{2 g-1}<a_{2 g-1}^{(n)}$. Let $\phi_{n} \in B(L, \Gamma)$ be the Teichmüller point of $T(\Gamma)$ associated with $S_{n}$.

We have the following

THEOREM 1. Under the above deformation, that is, if $a_{2 g-1}^{(n)}$ tends to $\infty$, then $\phi_{n}$ goes to a cusp on $\partial T(\Gamma)$ as $n \rightarrow \infty$.

For brevity, we consider the case of genus 2, that is, $S$ is a hyperelliptic surface with branch points $a, b, 0,1, c, \infty$ on $R(a<b<0<1<c$ and $c>|a|)$ and branch cuts of the segments on the real axis joining $a$ to $b, 0$ to 1 and $c$ to $\infty$ (see Fig. 2).

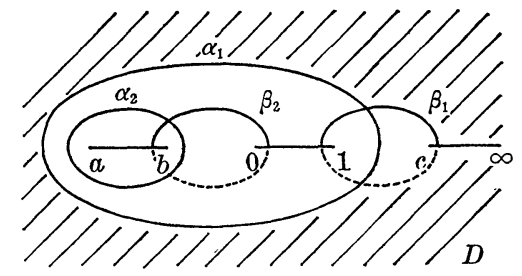

Fig. 2.

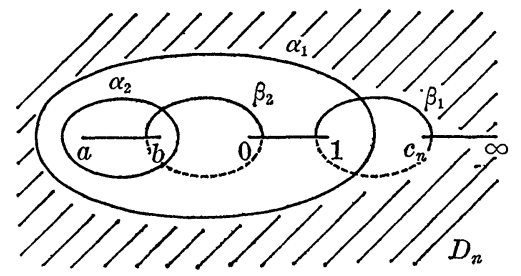

Fig. 3

Let $S_{n}$ be the hyper-elliptic surface with branch points $a, b, 0,1, c_{n}, \infty$ $\left(c<c_{n}\right)$ as in the above Fig. 3. Take a Fuchsian group $\Gamma$ such that $U / \Gamma=S$ and consider the deformation under which $S$ tends to $S_{n}$. Give a homology basis $\alpha_{1}, \alpha_{2}, \beta_{1}, \beta_{2}$ on $S$ as in the above Fig. 2. Especially let $\alpha_{1}$ be a circle of the radius $r$ with center at $0,|a|<r<c$. On the another sheet we denote by $\alpha_{1}^{\prime}$ the circle with the same projection as $\alpha_{1}$. Let $D$ be the ring domain bounded by $\alpha_{1}$ and $\alpha_{1}^{\prime}$ (the shaded part in Fig. 2 is the half of the domain). Furthermore we write the same homology basis $\alpha_{1}, \alpha_{2}, \beta_{1}, \beta_{2}$ on $S_{n}$ and consider the same $\alpha_{1}$. Let $D_{n}$ be the ring domain bounded by $\alpha_{1}$ and $\alpha_{1}^{\prime}$ on $S_{n}$. To these loops $\alpha_{1}, \alpha_{2}, \beta_{1}, \beta_{2}$ on $S$ we assign Möbius transformations $A_{1}, A_{2}, B_{1}, B_{2}$ in $\Gamma$, respectively.

We construct a q.c. mapping $f_{n}: S \rightarrow S_{n}$ as follows. Let $\Phi$ be

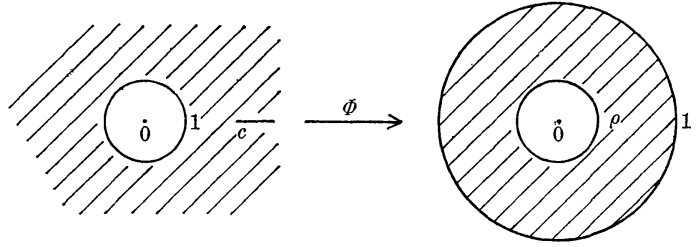

Fig. 4. 
the conformal mapping of the Grötzsch extremal region to the annulus $\{0<\rho<|w|<1\}$ (see Fig. 4). Then $\Phi(c)=1 / \rho$ (Künzi*). We map $D$ and $D_{n}$ to the annuli $K:\{0<\rho<|z|<1\}$ and $K_{n}:\left\{0<\rho_{n}<|z|<1\right\}$ conformally, respectively, where the $r$ is the radius of the circle $\alpha$. Then

$$
\Phi((1 / r) c)=1 / \sqrt{\rho} \text { and } \Phi\left((1 / r) c_{n}\right)=1 / \sqrt{\rho_{n}} .
$$

We define a q.c. mapping $\tilde{f}_{n}: K \rightarrow K_{n}$ by setting

$$
\tilde{f}_{n}\left(\sigma e^{i \theta}\right)=\sigma_{n} e^{i \theta}, \quad 0 \leq \theta \leq 2 \pi, \rho \leq \sigma \leq 1,
$$

where $\sigma_{n}=\left(\left(\rho_{n}-1\right) /(\rho-1)\right)(\sigma-1)+1$. The mapping $\tilde{f}_{n}$ has the property such that $\tilde{f}_{n}\left(\rho e^{i \theta}\right)=\rho_{n} e^{i \theta}$ on $|z|=\rho$ and $\tilde{f}_{n}\left(e^{i \theta}\right)=e^{i \theta}$ on $|z|=1$. Set $h(z)=(1 / r) z$ and $g=\Phi \circ h$. Now we define a q.c. mapping $f_{n}^{*}: D$ $\rightarrow D_{n}$ by setting $f_{n}^{*}=g^{-1} \tilde{f}_{n} g$. Then it is easily seen that $f_{n}^{*}=$ id. on $\alpha_{1}$ and $\alpha_{1}^{\prime}$. We define the q.c. mapping $f_{n}: S \rightarrow S_{n}$ by setting

$$
f_{n}= \begin{cases}f_{n}^{*} & \text { on } D \\ \text { id. } & \text { on } S-D .\end{cases}
$$

LEMMA 1. $\lim _{n \rightarrow \infty} \rho_{n}=0$ if and only if $\lim _{n \rightarrow \infty} c_{n}=\infty$.

Proof. By Künzi it holds

$$
r / 4 c_{n}<\sqrt{\rho_{n}}<r\left(2 c_{n}-r\right) /\left(8 c_{n}\left(c_{n}-r\right)\right) .
$$

Using the above inequalities we immediately have the desired result.

LEMMA 2. For $f_{n}$ constructed above, there uniquely exist a q.c. mapping $F_{n}$ and the natural projection $\pi_{n}: F_{n}(U) \rightarrow S_{n}$ which satisfy the following conditions.

1) $\pi_{n} F_{n}=f_{n} \pi$

2) if we set $G_{n}=F_{n} \Gamma F_{n}^{-1}$, then $F_{n}(U) / G_{n}=S_{n}$

3) $F_{n}(0,1, \infty)=(0,1, \infty)$,

where $\pi$ denote the natural projection from $U$ onto $S$.

Proof. Define a Beltrami differential $\mu_{n}$ on $S$ by setting $\mu_{n}=\left(f_{n}\right)_{z}$ $/\left(f_{n}\right)_{z}$. Lifting $\mu_{n}$ to a fundamental set $\omega_{0}$ for $\Gamma$ in $U$ and denote it by $\tilde{\mu}_{n}$, that is,

$$
\tilde{\mu}_{n}(z)=\mu_{n}(\pi z) \overline{\pi^{\prime}(z)} / \pi^{\prime}(z), \quad z \in \omega_{0} .
$$

*) H. P. Künzi, Quasikonforme Abbildungen, in Ergebnisse der Mathematik, Springer Verlag, Berlin, 1960. 
Then we extend $\tilde{\mu}_{n}$ over the whole of $U$ by the functional equation

$$
\tilde{\mu}_{n}(A z) \overline{A^{\prime}(z)} / A^{\prime}(z)=\tilde{\mu}_{n}(z), \quad z \in \omega_{0}, A \in \Gamma .
$$

We set $\tilde{\mu}_{n}(z)=0$ in $\hat{C}-U$. We denote the extended Beltrami coefficient by $\tilde{\mu}_{n}^{*}$. Using the well-known result due to Ahlfors and Bers, there uniquely exists a q.c. mapping $F_{n}$ such that $\left(F_{n}\right)_{\bar{z}}=\tilde{\mu}_{n}^{*}\left(F_{n}\right)_{z}$ and $F_{n}(0,1, \infty)=(0,1, \infty)$. This is the desired mapping. For, we set $G_{n}$ $=F_{n} \Gamma F_{n}^{-1}$ and define $\pi_{n}$ as follows. For any $w \in F_{n}(U)$, there uniquely exists $z \in U$ such that $F_{n}(z)=w$. Setting $\pi(z)=\zeta$ and $f_{n}(\zeta)=\omega$, we define $\pi_{n}$ as $\pi_{n}(w)=\omega$. This definition is well-defined, since it is easily seen that if we set $w^{\prime}=A w$ for any $A \in G_{n}$, then $\pi_{n}\left(w^{\prime}\right)=\omega$. Our proof is now complete.

Set $F_{n} \mid L=W_{n}$ and $\phi_{n}=\left\{W_{n}, z\right\}, z \in L$. Then $\phi_{n} \in B(L, \Gamma)$. If we set $G_{n}=F_{n} \Gamma F_{n}^{-1}$, then $G_{n}$ is a quasi Fuchsian group. We assign to the loop $\alpha$ on $S$ a Möbius transformation $A \in \Gamma$. Set $A_{n}=F_{n} A F_{n}^{-1}$. Let the multipliers of $A$ and $A_{n}$ be $\lambda$ and $\lambda_{n}$, respectively. Without loss of generality, we may assume that $A(z)=\lambda z$ and $A_{n}(z)=\lambda_{n} z$.

LEMMA 3. If $\lim _{n \rightarrow \infty} c_{n}=\infty$, then $\lim _{n \rightarrow \infty} \log \left|\lambda_{n}\right|=0$.

Proof. We denote by $C$ the set of all simple closed rectifiable curves $c$ separating 0 and $\infty$ and denote by $M_{n}$ the extremal length of $C$ on $F_{n}(\hat{C}) /\left\{A_{n}\right\}$, that is,

$$
M_{n}=\sup _{\sigma} \frac{\left(\inf _{c} \int_{c} \sigma(z)|d z|\right)^{2}}{\iint_{F_{n}(\hat{c}) /\left\{A_{n}\right\}} \sigma(z)^{2} d x d y}
$$

where $\left\{A_{n}\right\}$ is a cyclic group generated by $A_{n}$ and $\sigma(z)$ is a non-negative measurable function satisfying the identity $\left|\lambda_{n}\right| \sigma\left(\lambda_{n} z\right)=\sigma(z)$. Then it is known by Bers [3] that

$$
M_{n}=2 \pi / \log \left|\lambda_{n}\right| \text {. }
$$

We denote by $\ell_{n}$ the lifting, joining 0 and $\infty$, of the branch cut $\left(c_{n}, \infty\right)$ and by $\tilde{D}_{n}$ the lifting, containing $\ell_{n}$, of the ring domain $D_{n}$ to the complement of $W_{n}(L)$. We denote by $C_{n}^{*}$ the set of all curves joining the boundary $|z|=1$ to the another boundary $|z|=\rho_{n}$ in the annulus $K_{n}$ and denote by $M_{n}^{*}$ the extremal length of $C_{n}^{*}$ in $K_{n}$. It is easily seen that 


$$
M_{n}^{*}=-\log \rho_{n} / 2 \pi .
$$

For any curve $c \in C_{n}$, there exists a curve $\tilde{c}^{*}$ in $\tilde{D}_{n}$ such that $\tilde{c}^{*}$ is a part of $c$ and a lifting of some $c^{*} \in C_{n}^{*}$. Then from the well-known fact, we have

$$
M_{n} \geqq M_{n}^{*} .
$$

By Lemma 1, if $\lim _{n \rightarrow \infty} c_{n}=\infty$, then $\lim _{n \rightarrow \infty} \rho_{n}=0$. Hence from (5), (6) and (7), we have the desired result. Our proof is now complete.

Proof of Theorem 1. Denote by $\tau_{n}$ the trace of $A_{n}$, then there is a relation

$$
\tau_{n}^{2}=\lambda_{n}+1 / \lambda_{n}+2 .
$$

By Lemma 3 and a simple computation, we have $\lim _{n \rightarrow \infty} \tau_{n}^{2}=4$. Hence $A_{0}$, the limit of $A_{n}$, is parabolic or the identity. Since by Bers [3], the mapping $\theta_{\phi}: \Gamma \rightarrow G_{0}$ is isomorphic, $A_{0}$ is not the identity, where $G_{0}$ is the limit of sequence of $G_{n}$ associated with $\left\{\phi_{n}\right\}$. Hence $\phi_{0}$, the limit of $\phi_{n}$, is cusp. The existence of $\phi_{0}$ follows from the fact that $\overline{T(T)}$, the closure of $T(\Gamma)$, is a compact set. Our proof is now complete.

Remark. (1) In Theorem 1 we considered only the case where $a_{2 g-1}$ goes to $\infty$ and $a_{j}(j=1, \cdots, 2 g-2)$ and the cuts joining $a_{2 j-1}$ to $a_{2 j}$ $(j=1, \cdots, g-1)$ are fixed, but we have a similar result to Theorem 1 even in the case when $a_{2 j-1}$ goes to $a_{2 j}(j=1, \cdots, g-1)$.

(2) We only considered cusps for " $\alpha$ "-cycles, but the same results is valid for " $\beta$ "-cycles.

Next we consider the case where $\Gamma$ is a finitely generated Fuchsian group of the first kind such that $U / \Gamma$ is a general compact Riemann surface, and denote by $T(\Gamma)$ the Teichmüller space attached to $\Gamma$. Let $C_{1}, \cdots, C_{n}$ be homotopically independent loops on the Riemann surface $U / \Gamma=S$, that is, roughly speaking they are $n$ disjoint simple loops on $S$ no two of which are freely homotopic (as in Maskit [7]). We call a "pinching" deformation the operation which squeezes some $C_{j}$ down to a point. Then we have the following by a similar proof to the above one.

THEOREM 1'. Under a "pinching" deformation, there exists a sequence $\left\{\phi_{n}\right\} \in T(\Gamma)$ tending to a cusp on $\partial T(\Gamma)$. 
Remark. This theorem contains Theorem 1 and was proved by a different method by Bers [3].

\section{Cusps obtained by the "pinching and twisting" deformations.}

In this section we shall consider cusps on the boundary of Teichmüller space $T(\Gamma)$ obtained by the "pinching and twisting" deformations.

Take homotopically independent loops $\alpha_{1}, \cdots, \alpha_{n}$ on $S$ and let $D_{1}, \cdots, D_{n}$ be ring domains which are disjoint each other and assume that each $D_{j}$ contains $\alpha_{j}(j=1, \cdots, n)$. For brevity let $n=1$. For the case of $n \geqq 2$, we can similarly treat to the case of $n=1$. Let $A$ be an element in $\Gamma$ associated with the loop $\alpha_{1}$. We write $D$ and $\alpha$ instead of $D_{1}$ and $\alpha_{1}$, respectively. We will study how the corresponding Teichmüller points vary under the following "pinching and twisting" deformation for $D$.

We will construct the Riemann surface $S_{n}$ from $S$ as follows. Let $D$ map to the annulus $K:\{0<\rho<|z|<1\}$ conformally such that the image of $\alpha$ is homotopic to the circle $|z|=\sqrt{\rho}$ in $K$. We denote again by the same letter $\alpha$ the inverse image of $|z|=\sqrt{ } \bar{\rho}$. Let $K^{1}$ and $K^{2}$ be the annuli $\{0<\sqrt{\rho}<|z|<1\}$ and $\{0<\rho<|z|<\sqrt{\rho}\}$, respectively. The inverse images in $D$ of $K^{1}$ and $K^{2}$ are denoted by $D^{1}$ and $D^{2}$, respectively. By the q.c. mapping $\tilde{f}_{n}$ defined later, the annuli $K^{1}$ and $K^{2}$ are mapped onto the annuli $K_{n}^{1}:\left\{0<\sqrt{\rho_{n}}<|w|<1\right\}$ and $K_{n}^{2}:\left\{0<\rho_{n}<|w|<\sqrt{\rho_{n}}\right\}$, respectively as follows, where $\rho_{n}=\exp \left(-\sqrt{4 \theta_{n}^{2}+(\log \rho)^{2}}\right),-\infty<\theta_{n}<$ $+\infty$. We define $\tilde{f}_{n}$ by setting

$$
\tilde{f}_{n}(z)=\left\{\begin{array}{l}
\exp \left\{\left(\log \rho_{n} / \log \rho\right) \xi+i\left(\eta-\left(2 \theta_{n} / \log \rho\right) \xi\right)\right\} \quad \text { in } K^{1} \\
\exp \left\{\left(\log \rho_{n} / \log \rho\right) \xi+i\left(\eta-2 \theta_{n}+\left(2 \theta_{n} / \log \rho\right) \xi\right)\right\} \quad \text { in } K^{2},
\end{array}\right.
$$

where $z=e^{\xi+i \eta},-\infty<\xi \leqq 0,-\infty<\eta<+\infty$. That is, the mapping $\tilde{f}_{n}$ maps the logarithmic spiral joining $e^{i \theta}$ to $\sqrt{\rho} e^{i\left(\theta+\theta_{n}\right)}$ in $K^{1}$ to the segment joining $e^{i \theta}$ to $\sqrt{\rho_{n}} e^{i \theta}$ in $K_{n}^{1}$ and maps the logarithmic spiral joining $\sqrt{\rho} e^{i\left(\theta+\theta_{n}\right)}$ to $e^{i \theta}$ in $K^{2}$ to the segment joining $\sqrt{\rho_{n}} e^{i \theta}$ to $\rho_{n} e^{i \theta}$ in $K_{n}^{2}$. Hence $\tilde{f}_{n}$ maps $e^{i \theta}$ and $\rho e^{i \theta}$ to $e^{i \theta}$ and $\rho_{n} e^{i \theta}$, respectively (see Fig. 5).

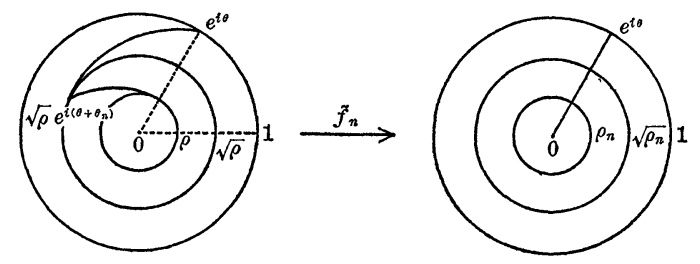

Fig. 5. 
We denote by $S_{n}$ the Riemann surface obtained from $S$ with the following boundary correspondence by replacing $D$ by the annulus $D_{n}$, where $D_{n}$ is a domain conformally equivalent to the annulus $\left\{0<\rho_{n}<\right.$ $|w|<1\}$. The boundary points $e^{i \theta}$ and $\rho_{n} e^{i \theta}$ of $D_{n}$ are to correspond to the same points of $S$ as the boundary points $e^{i \theta}$ and $\rho e^{i \theta}$ of $D$, respectively. We denote by $g$ and $g_{n}$ the conformal mappings constructed above from $D$ to the annulus $\{0<\rho<|z|<1\}$ and from $D_{n}$ to the annulus $\left\{0<\rho_{n}<|w|<1\right\}$, respectively.

Let $f_{n}: S \rightarrow S_{n}$ be a q.c. mapping which is the identity on $S-D$ and $g_{n}^{-1} \tilde{f}_{n} g$ in $D$. Then we obtain the following by a similar method to the proof of Lemma 2.

LEMma 4. There uniquely exist a q.c. mapping $F_{n}$ of $\hat{\boldsymbol{C}}$ to $\hat{\boldsymbol{C}}$ and the natural projection $\pi_{n}: F_{n}(U) \rightarrow S_{n}$ which satisfy the following conditions :

1) $\pi_{n} F_{n}=f_{n} \pi$

2) if we set $F_{n} \Gamma F_{n}^{-1}=G_{n}$, then $F_{n}(U) / G_{n}=S_{n}$

3) $F_{n}(0,1, \infty)=(0,1, \infty)$,

where $\pi: U \rightarrow S$ is the natural projection.

Setting $F_{n} \mid L=W_{n}$ and $\left\{W_{n}, z\right\}=\phi_{n}$, then $\phi_{n} \in B(L, \Gamma)$. We denote by $\lambda$ the multiplier of $A$ associated with the loop $\alpha$ and denote by $\lambda_{n}$ the multiplier of $A_{n}=F_{n} A F_{n}^{-1} \in G_{n}$.

LEMMA 5. If $\lim _{n \rightarrow \infty} \theta_{n}= \pm \infty$, then $\lim _{n \rightarrow \infty} \log \left|\lambda_{n}\right|=0$.

Proof. As the same in the proof of Lemma 3, let $C$ be the set of all rectifiable simple closed curves separating 0 and $\infty$ and let $M_{n}$ be the extremal length of $C$ on $F_{n}(\hat{C}) /\left\{A_{n}\right\}$. Then we obtain

$$
M_{n}=2 \pi / \log \left|\lambda_{n}\right| \text {. }
$$

Let $\tilde{C}_{n}$ be the set of all curves joining one boundary $|z|=1$ to the another one $|z|=\rho_{n}$ in $\tilde{K}_{n}=\left\{0<\rho_{n}<|z|<1\right\}$ and let $\tilde{M}_{n}$ be the extremal length of $\tilde{C}_{n}$ in $\tilde{K}_{n}$. Then we have

$$
\tilde{M}_{n}=\left|\log \rho_{n}\right| / 2 \pi \text {. }
$$

Noting that if $\lim _{n \rightarrow \infty} \theta_{n}= \pm \infty$, then $\lim _{n \rightarrow \infty}\left|\log \rho_{n}\right|=+\infty$, we have

$$
\lim _{n \rightarrow \infty} \tilde{M}_{n}=+\infty \text {. }
$$


We denote by $\tilde{K}_{n}^{*}$ a connected lifting whose closure contains 0 and $\infty$ in $F_{n}(U)$ of $\tilde{K}_{n}$ and by $\tilde{C}_{n}^{*}$ the lifting of $\tilde{C}_{n}$ in $\tilde{K}_{n}^{*}$, respectively. Then for any $c \in C$, there is a $\tilde{c}^{*} \in \tilde{C}_{n}^{*}$ such that $\tilde{c}^{*}$ is a part of $c$. Then from the well-known fact, we have

$$
M_{n} \geqq \tilde{M}_{n}
$$

Through from (8) to (11), we have the desired result, that is, $\lim _{n \rightarrow \infty} M_{n}$ $=+\infty$ so $\lim _{n \rightarrow \infty} \log \left|\lambda_{n}\right|=0$. Our proof is now complete.

We have the following by the same method as in the proof of of Theorem 1.

THEOREM 2. Let $\Gamma$ be a finitely generated Fuchsian group of the first kind such that $U / \Gamma$ is a compact Riemann surface of genus $g \geqq 2$. Let $\alpha_{1}, \cdots, \alpha_{n}$ be homotopically independent loops on $S$ as defined above. Then under the above "pinching and twisting" deformation, there is a sequence $\left\{\phi_{n}\right\}$ of Teichmüller points such that $\phi_{n}$ goes to a cusp on the boundary $\partial T(\Gamma)$.

\section{REFERENCES}

[1] L. V. Ahlfors, Finitely generated Kleinian groups, Amer. J. Math., 86 (1964), $413-429$ and 87 (1965), 759.

[ 2 ] — Lectures on quasiconformal mappings (Van Nostrand, New York), 1966.

[ 3 ] L. Bers, On boundaries of Teichmüller spaces and on Kleinian groups: I, Ann. of Math., 91 (1970), 570-600.

[4] — , Spaces of Kleinian groups, Lecture notes in Mathematics, 155 (1970), Springer, Berlin, 9-34.

[ 5 ] C. I. Kalme, Remarks on a paper by Lipman Bers, Ann. of Math., 91 (1970), 601-606.

[ 6 ] I. Kra, On spaces of Kleinian groups, Comment. Math. Helv., 47 (1972), 53-69.

[ 7 ] B. Maskit, On boundaries of Teichmüller spaces and on Kleinian groups: II, Ann. of Math., 91 (1970), 607-639.

[ 8 ] — Self-maps of Kleinian groups, Amer. J. Math., 93 (1971), 840-856.

Department of Mathematics

Faculty of Science

Shizuoka University 